\title{
Active compounds and distinctive sensory features provided by American ginseng (Panax quinquefolius $L$.) extract in a new functional milk beverage
}

\author{
A. Tárrega, ${ }^{*}$ A. Salvador, ${ }^{*}$ M. Meyer,† N. Feuillère,† A. Ibarra,‡ M. Roller,§ D. Terroba,\# C. Madera,\# \\ J. R. Iglesias,\# J. Echevarría,\# and S. Fiszman*1 \\ *Instituto de Agroquímica y Tecnología de Alimentos (IATA), Consejo Superior de Investigaciones Científicas (CSIC), Agustín Escardino, \\ 7, 46980 Paterna (Valencia), Spain \\ †Naturex Spain SL, Autovia A3, salida 343, Camino de Torrent s/n, 46930 Quart de Poblet, Spain \\ $\ddagger$ Naturex Inc., 375 Huyler St., South Hackensack, NJ 07606 \\ §Naturex SA, Site d'Agroparc BP 1218, 84911 Avignon Cedex 9, France \\ \#Corporación Alimentaria Peñasanta SA (CAPSA), Sierra de Granda, s/n, 33199-Siero, Asturias, Spain
}

\begin{abstract}
American ginseng (Panax quinquefolius L.) has recognized neurocognitive effects, and a ginsenoside-rich extract of the root of the plant has been shown to improve cognitive functions in young adults. This study aimed at assessing the chemical and sensory profiles of a UHT-treated, low-lactose functional milk containing American ginseng. Individual ginsenosides in the milk were analyzed by HPLC. Descriptive sensory analysis was performed by a trained panel to quantitatively document sensory changes resulting from the addition of ginseng and the UHT process on flavored and unflavored milks. Consumer acceptance of the product was also investigated. Total ginsenoside content in the UHT-treated milk enriched with the ginseng extract after UHT process treatment was $7.52 \mathrm{mg} / 100 \mathrm{~g}$ of milk, corresponding to a recovery of $67.6 \%$ compared with the content in the unprocessed extract. The intake of 150 to $300 \mathrm{~mL}$ of this ginseng-enriched milk provides the amount of total ginsenosides (11.5 to 23 $\mathrm{mg}$ ) necessary to improve cognitive function after its consumption. Both the presence of ginsenosides and their thermal treatment affected some sensory properties of the milk, most notably an increase in bitterness and metallic taste, the appearance of a brownish color, and a decrease in milky flavor. Levels of brown color, bitterness, and metallic taste were highest in the industrially processed ginseng-enriched milk. The bitterness attributable to ginseng extract was reduced by addition of vanilla flavor and sucralose. A consumer exploratory study revealed that a niche of consumers exists who are willing to consume this type of product.
\end{abstract}

Key words: UHT-treated milk, ginsenoside, sensory property, consumer acceptance

Received January 13, 2012.

Accepted April 13, 2012.

${ }^{1}$ Corresponding author: sfiszman@iata.csic.es

\section{INTRODUCTION}

American ginseng (Panax quinquefolius L. syn. Panax quinquefolium L.) has long been recognized for its effects on the central nervous system, such as its enhancement of cognitive performance and mood (Attele et al., 1999; Qi et al., 2011) and its benefits in aging, central nervous system disorders, and neurodegenerative diseases (Christensen, 2009). In humans, a standardized American ginseng ( $P$. quinquefolius L.) extract, containing 13 to $20 \%$ ginsenosides, was shown to improve memory in young healthy volunteers (Sutherland et al., 2010), corroborating previous findings (Kennedy and Scholey, 2003) with Asian ginseng (Panax ginseng L.). The chief active constituents in ginseng are triterpenoid saponins, known as ginsenosides (Jia and Zhao, 2009), which are responsible for most of its bioactivity. Typically, American ginseng contains 40 to $60 \mathrm{~g} / \mathrm{kg}$ of total ginsenosides [4 to 6\% (wt/wt); Qi et al., 2011], of which Rb1, Rb2, $\mathrm{Rc}, \mathrm{Rd}, \mathrm{Re}$, and Rg1 are the principal ginsenosides (Ren and Chen, 1999a).

Cereboost (Naturex Inc., South Hackensack, NJ) is a native extract of American ginseng (P. quinquefolius L.) roots that has been standardized to a total ginsenoside content of 10 to $12 \%$. This American ginseng extract was recently shown in a randomized, double-blind, placebo-controlled, crossover trial to enhance working memory, attention (choice reaction time accuracy), secondary memory (immediate word recall accuracy), and mood in healthy young individuals across a range of dosages (100, 200, and $400 \mathrm{mg} / \mathrm{d}$; Scholey et al., 2010) - the first demonstration of the beneficial effects of this ginseng extract on cognitive function.

With regard to regulatory oversight, American ginseng has a generally recognized as safe (GRAS) status in the United States, has been used extensively as an ingredient in North America, and is not classified as a novel food in the European Union Novel Food catalog. The UHT-treated milk with American ginseng that 
we report is registered in Belgium under NUT number 1462/10, issued on December 16, 2011.

Ginseng beverages, such as infusions, coffees, teas, and energy drinks, are popular in the Asian market. However, in a recent study on consumers' attitudes and expectations of ginseng food products in the United States (Chung et al., 2011), consumers reported low initial interest in this type of ginseng product from the Asian market. According to Tamamoto et al. (2010a), the challenges of incorporating ginseng into food products are 2-fold: retaining the healthful properties of ginseng and minimizing its bitter taste.

In the dairy industry, interest in introducing milk and yogurt products with health benefits has been growing, particularly the interest in products that increase performance or boost immunity (Jäger et al., 2010), but dairy products that are enriched with $P$. quinquefolius L. extract have not been reported. Under the aegis of the SENIFOOD (food for seniors) project, which is devoted to designing food products for a suitable and balanced diet in elderly Spanish people, the aim of this work was to assess the feasibility of incorporating a ginsenoside-rich American ginseng extract into low-lactose milk by an industrial process, to obtain a drink that improves cognitive functions in the elderly. The ginsenoside content and sensory characteristics of the resulting product are examined.

\section{MATERIALS AND METHODS}

\section{Materials}

The American ginseng extract Cereboost (Naturex; Reference No. GA505000) used was obtained through an industrial process, as described in Scholey et al. (2010). Raw milk was obtained from farms in northern Spain (transport temperature $<8^{\circ} \mathrm{C}$ ). Vanilla flavor mix was purchased from Condetta (Condetta $\mathrm{GmbH} \& \mathrm{Co}$. KG, Halle, Germany), powdered sucralose (Splenda) was obtained from Tate \& Lyle (Madrid, Spain), and lactase enzyme (Ha-Lactase 5200) was sourced from Christian Hansen, (Tres Cantos, Madrid, Spain).

\section{Industrial Processing of Milk Samples}

The industrial process by which ginseng-enriched flavored milk was produced was carried out at Corporación Alimentaria Peñasanta SA (CAPSA, Asturias, Spain). Raw milk was pooled, heated to 60 to $65^{\circ} \mathrm{C}$ for 15 to $20 \mathrm{~s}$ to reduce the initial microbial count, and passed through a centrifugal separator (GEA Westfalia Separator, Oelde, Germany). Somatic cells and other exogenous particles from milk with higher density were removed during this step. The cream was separated from the skim milk using the same centrifugal system. An appropriate amount of cream was then added back to the recovered skim milk to adjust the fat content to $1 \%$ (wt/vol). Lactase [0.1\% (wt/vol)] was subsequently added to partially digest the lactose and to obtain a more digestible milk. The residual lactose content was determined to be $0.21 \%$ (wt/vol).

The American ginseng extract was added to the standardized low-lactose milk at a concentration of $0.1 \%$ (wt/vol), providing 10 to $12 \mathrm{mg}$ of ginsenosides/100 $\mathrm{mL}$ milk. Other ingredients, such as sweeteners and flavors, were added to improve the sensory properties of the product. The nutritional composition of the milk enriched with ginseng extract is presented in Table 1. After the ingredients were mixed, the product was sterilized by direct UHT treatment, wherein the milk was heated quickly to 148 to $150^{\circ} \mathrm{C}$ for 6 to $7 \mathrm{~s}$ by direct steam injection and cooled immediately to $80^{\circ} \mathrm{C}$ to $85^{\circ} \mathrm{C}$ through flash vacuum evaporation of the water that condensed from the steam; a subsequent homogenization step $\left(200 \mathrm{~kg} / \mathrm{cm}^{2}\right)$ was also performed under aseptic conditions. The milk was cooled to room temperature (22 to $25^{\circ} \mathrm{C}$ ) and packaged under aseptic conditions into sterilized Tetra Pak packages (Tetra Pak International SA, Pully/Lausanne, Switzerland).

\section{Analysis of Ginsenosides Content}

All reagents were analytical grade and purchased from Scharlau (Scharlau Chemie SA, Barcelona, Spain) unless otherwise stated. The following method was developed by Naturex Spain SL (Quart de Poblet, Spain) for the extraction, purification, and quantification of ginsenosides in the milk matrix. Sixty milliliters of the milk enriched with American ginseng extract was measured carefully and adjusted to $\mathrm{pH} 4$ with acetic acid $(99 \%)$ to precipitate the casein fraction. The solu-

Table 1. Nutritional composition for $100 \mathrm{~mL}$ of milk enriched with American ginseng extract ${ }^{1}$

\begin{tabular}{ll}
\hline Nutritional fact & Value per $100 \mathrm{~mL}$ \\
\hline Energy & $41 \mathrm{kcal}(172 \mathrm{~kJ})$ \\
Proteins & $3.1 \mathrm{~g}$ \\
Carbohydrates & $4.7 \mathrm{~g}$ \\
Sugars & $4.7 \mathrm{~g}$ \\
Fat & $1 \mathrm{~g}$ \\
Saturated fat & $0.69 \mathrm{~g}$ \\
Unsaturated fat & $0.31 \mathrm{~g}$ \\
Fiber & $0 \mathrm{~g}$ \\
Sodium & $0.06 \mathrm{~g}$ \\
Calcium & $120 \mathrm{mg}\left(15 \% \mathrm{RDI}^{2}\right)$ \\
Ginsenosides & $10 \mathrm{to} 12 \mathrm{mg}$ \\
Lactose & $0.21 \mathrm{~g}$ \\
\hline
\end{tabular}

${ }^{1}$ Cereboost (Naturex Inc., South Hackensack, NJ).

${ }^{2} \mathrm{RDI}=$ recommended daily intake. 
tion was centrifuged at $3,000 \times g$ for $12 \mathrm{~min}$ at 22 $\pm 2^{\circ} \mathrm{C}$ (Orto Alresa, Madrid, Spain), the supernatant was collected, and methanol was added ( $30 \%$ of supernatant volume) to precipitate globulins. The solution was centrifuged again at $3,000 \times g$ for 12 min at 22 $\pm 2^{\circ} \mathrm{C}$, and the supernatant was evaporated under a vacuum at $60^{\circ} \mathrm{C}$ on a rotary evaporator (Rotavapor, R II, Büchi, Flawil, Switzerland). The evaporate was carefully set aside.

Precipitated proteins were further extracted with $200 \mathrm{~mL}$ of methanol, homogenized on a disperser (UltraTurrax, IKA, Staufen im Breisgau, Germany), and filtered through a 42.5-mm-diameter qualitative filter paper (Albet-Hahnemuehle SLU, Barcelona, Spain) using a Büchner funnel. The filtrate was combined with the evaporated supernatant and evaporated to dryness on a rotary evaporator as described above. The residue was solubilized in $10 \mathrm{~mL}$ of HPLC diluent [water:acetonitrile:ethanol, 60:20:20 (by vol)], and the resulting solution was extracted with $25 \mathrm{~mL}$ of chloroform in a water bath $\left(30^{\circ} \mathrm{C}, 10 \mathrm{~min}\right)$ to remove fat. After centrifugation, the ginsenoside-containing supernatant was adjusted to $10 \mathrm{~mL}$ with HPLC diluent before HPLC analysis.

The identification and quantification of ginsenosides were performed per the Determination of Ginsenosides in Panax Ginseng and American Ginseng by HighPerformance Liquid Chromatography by the National Sanitation Foundation (INA Method 103.000; National Sanitation Foundation, 2012), per Li et al. (1996). The HPLC system consisted of an Agilent 1100 series HPLC instrument (Agilent, Torrance, CA) that was equipped with an Agilent 1100 autosampler and UV detector (203 $\mathrm{nm})$. The milk containing ginseng extract, or the ginseng extract or a ginsenoside standard solution $(20 \mu \mathrm{L})$ was injected into an ACE C18 column $(5 \mu \mathrm{m}, 4.6 \mathrm{~mm}$ i.d. $\times 250 \mathrm{~mm}$, Advanced Chromatography Technologies, Aberdeen, Scotland). The mobile phase consisted of HPLC-grade acetonitrile (A) and $0.1 \%$ phosphoric acid in water (B; Synergy Water Purification System, Millipore, Molsheim, France).

The following gradient program, including changes in solvent, was applied: a solution of $21 \% \mathrm{~A}$ and $79 \%$
B was maintained for 20 min, followed by a linear gradient to $35 \% \mathrm{~A}$ and $65 \% \mathrm{~B}$ in $40 \mathrm{~min}$, which was maintained for 5 min and increased to $90 \% \mathrm{~A}$ and $10 \%$ $\mathrm{B}$ in $5 \mathrm{~min}$ (total run time, $70 \mathrm{~min}$ ). The system was then reequilibrated to the initial composition for 10 min. Flow rate was maintained at $1.2 \mathrm{~mL} / \mathrm{min}$, and the column temperature was set to $20^{\circ} \mathrm{C}$.

Identification of individual ginsenosides was performed at $203 \mathrm{~nm}$, and the quantity of each ginsenoside was calculated by comparing the peak area obtained with that of external standards. As references, external standards (ginsenosides $\mathrm{Rg}_{1}$, Re, Rf, $\mathrm{Rb}_{1}, \mathrm{Rc}, \mathrm{Rb}_{2}$, and Rd; Extrasynthese, Genay, France) and the American ginseng extract (Cereboost, 1,000 mg) were dissolved in $50 \mathrm{~mL}$ of HPLC diluent [water:acetonitrile:ethanol, 60:20:20 (by vol)] before injection into the chromatograph for ginsenoside analysis.

\section{Sensory Evaluation Samples}

The effects of ginseng extract on the sensory properties of unflavored and flavored milk samples, and the sensory characteristics of UHT-treated milk with ginseng extract were examined with regard to the sensory profile of 5 samples whose characteristics are described in Table 2.

Samples A, B, and C were prepared in the laboratory in batches of $1,000 \mathrm{~g}$, using the control sample (low-lactose milk) as the basis for each formulation. The ingredients (milk, vanilla, sucralose, and ginseng extract) were weighed in a flask and mixed on a magnetic stirrer (Selecta, Barcelona, Spain) for $20 \mathrm{~min}$. The samples were refrigerated $\left(4^{\circ} \mathrm{C}\right)$ for $24 \mathrm{~h}$ before the sensory analysis. All samples (B, C, and D) contained the same amount of flavoring ingredients.

\section{Quantitative Descriptive Analysis}

The quantitative descriptive analysis (QDA) approach (Stone and Sidel, 1998) was chosen to identify and quantify the sensory properties of the 5 milk samples. Ten subjects served on the sensory panel. All

Table 2. Characteristics of the milk samples evaluated by the trained panel

\begin{tabular}{|c|c|}
\hline Sample & Description \\
\hline Control & Low-lactose milk prepared using the industrial process but without American ginseng extract, ${ }^{1}$ sucralose, or vanilla \\
\hline A & Milk (control) enriched with $0.1 \%$ ginseng extract \\
\hline B & Milk (control) with $0.04 \%$ vanilla aroma, and $0.001 \%$ sucralose \\
\hline $\mathrm{C}$ & Milk (control) with $0.1 \%$ ginseng extract, $0.04 \%$ vanilla aroma, and $0.001 \%$ sucralose \\
\hline $\mathrm{D}$ & Low-lactose milk prepared using the industrial process, with $0.1 \%$ ginseng extract, $0.04 \%$ vanilla aroma, and $0.001 \%$ sucralose \\
\hline
\end{tabular}

${ }^{1}$ Cereboost (Naturex Inc., South Hackensack, NJ). 
of them had good discrimination ability based on performance in preliminary triangle tests with basic tastes.

The QDA procedure included 2 steps: 1) the training of the panelists and 2) the final descriptive analysis of the samples. For the training, two 1-h sessions were devoted to generating the vocabulary that would be used to describe the sensory differences between milk samples. An initial list of descriptors that had been used by others (Tamamoto et al., $2010 \mathrm{~b}$ ) and 2 milk samples (the control and sample D) were presented to the panel. An open discussion established the final attributes and how they should be evaluated. The final list of these 10 descriptors, their end terms, and definitions are shown in Table 3. Four further 1-h sessions were used to train panelists in the use of a $10-\mathrm{cm}$ intensity scale for each attribute. Reference samples were used to fix the anchors to the scale. During each session, panelists evaluated the intensity of each attribute in some milk samples containing different concentrations of sucrose, vanilla, or ginseng extract, and the individual results were discussed to establish consensus criteria.

For the final descriptive analysis, the 5 samples (the control and samples A, B, C, and D) were tested in duplicate over 2 sessions. Each assessor evaluated the intensity of the selected attributes at individual booths in a standardized test room (ISO 8589; ISO, 2007). Samples $(30 \mathrm{~mL})$ were served in white plastic cups coded with random 3-digit numbers and presented monadically. Samples were scored for each attribute using the same scale as used during the training sessions. To reduce the influence of serving order, the samples evaluated in each session were served following a Williams design for 5 samples in balanced blocks for 10 assessors. Bread and mineral water were provided to cleanse the palate. Data acquisition was performed us- ing Compusense Five v.4.6 (Compusense Inc., Guelph, ON, Canada).

\section{Consumer Study}

An exploratory study with 100 participants was performed to evaluate consumer opinion of the ginsengenriched milk product. Consumers carried out evaluations in individual booths in a standardized test room (ISO 8589; ISO, 2007). Participants were first asked to indicate how much they would be willing to consume a "highly digestible semi-skimmed milk" and a "highly digestible semi-skimmed milk enriched with ginseng extract that improves cognitive function." The participants used 5-point scales from "I definitely would consume the product" to "I definitely would not consume it." Subsequently, they tasted and rated overall acceptability and purchase intention of the control milk and the UHT-treated milk enriched with ginseng extract (sample D). Samples $(30 \mathrm{~mL})$ were presented monadically with the above-mentioned information about the characteristics of each product. The order of samples presentation followed a balanced design for the 2 samples to avoid the influence of serving order. Acceptability was evaluated by using a 9-point hedonic scale ranging from 1 ("dislike extremely") to 9 ("like extremely"), and purchase intention was evaluated by using a 5-point scale of intention from "I definitely would buy this product" to "I definitely would not buy this product."

\section{Data Analysis}

For the QDA data, the concordance of the panel in the evaluation of each attribute was studied by con-

Table 3. List of attributes, descriptions, and end terms used during the evaluation sessions

\begin{tabular}{lll}
\hline Attribute & Description & End terms \\
\hline Color & $\begin{array}{l}\text { Magnitude of the difference in color perceived in the } \\
\text { product with respect to the white color of milk }\end{array}$ & White to beige \\
Sweet odor & $\begin{array}{l}\text { Odor perceived orthonasally with sweet, toasted, or floral } \\
\text { notes and also associated with burnt sugar }\end{array}$ & Weak to intense \\
Milk flavor & Flavor associated with fresh UHT whole milk & Weak to intense \\
Vanilla flavor & Sweet flavor, with toasted, floral, or spicy notes & Not perceived to intense \\
Metallic/root flavor & Flavor associated with the flavor of licorice root and some medicaments & Not perceived to intense \\
Sweetness & Describes the taste produced by aqueous solutions of substances such as sucrose & Weak to intense \\
Bitterness & Describes the taste produced by dilute aqueous solutions of & Not perceived to intense \\
Aftertaste & various substances such as quinine and caffeine & Weak to intense \\
Astringent & Taste sensation that appears in the mouth after the elimination of the product & Not perceived to intense \\
Viscosity & A mouth-drying sensation felt on the tongue and sides of the mouth & Thin to thick \\
& and produced by solutions of certain compounds such as tannins & Mechanical property perceived in the mouth related \\
\hline
\end{tabular}


sidering the sample $\times$ assessor interaction in a 2-way ANOVA (sample, assessor, sample $\times$ assessor). The contribution of each assessor to the significance of the interaction effect was examined using PanelCheck software (http://www.panelcheck.com). According to the PanelCheck program, 2 assessors showing disagreement with the rest of the panel in a high number of attributes were detected and their data were not considered further in the analysis. To study the significance of the effect of a sample, mixed model ANOVA was performed for each attribute, with assessors as random factors and samples as fixed factors (González-Tomás et al., 2009). Minimum significant differences were calculated using Tukey's test, with the significance level set at $P<0.05$. Principal components (PC) analysis was applied to the correlation matrix of the average values of the sensory attributes scores obtained for the milk samples. Both analyses were performed using the XLSTAT-Pro Version 2007 (Addinsoft, Paris, France). From the results of the consumer evaluation, the average acceptability values and standard deviations were calculated using the software XLSTAT-Pro Version 2007 (Addinsoft).

\section{RESULTS AND DISCUSSION}

\section{Ginsenoside Content in UHT-Treated Milk Enriched with Ginseng Extract}

Figure 1A shows the chromatogram of the ginseng extract, and Figure 1B shows the chromatogram of ginseng extract-enriched milk after the UHT treatment. The peak elution time of each ginsenoside was as follows: $\operatorname{Rg} 1$ at $18.3 \mathrm{~min}, \mathrm{Re}$ at $19.9 \mathrm{~min}, \mathrm{Rf}$ at $47.3 \mathrm{~min}$, $\mathrm{Rb} 1$ at $54.1 \mathrm{~min}, \mathrm{Rc}$ at $56.1 \mathrm{~min}, \mathrm{Rb} 2$ at $58.6 \mathrm{~min}$, and $\mathrm{Rd}$ at $63.4 \mathrm{~min}$.

The American ginseng extract had a total ginsenoside content of $11.12 \%$ (or $11.12 \mathrm{mg} / 100 \mathrm{mg}$ of ginseng extract), calculated as the sum of the contents of individual ginsenosides, as shown in Table 4. As expected, ginsenoside Rf was not detected (Harkey et al., 2001). Ginsenoside $\mathrm{Rf}$ is not present in American ginseng but exists in Asian ginseng ( $P$. ginseng L.); consequently, it is used as a marker to detect the adulteration of American ginseng.

The ginsenoside content of milk enriched with American ginseng extract was $7.52 \mathrm{mg}$ of total ginsenosides $/ 100 \mathrm{~g}$ of milk, corresponding to a recovery of $67.63 \%$ compared with the unprocessed ginseng extract sample (Table 4). The recovery of individual ginsenosides in milk with ginseng extract ranged from 57.4 to $100 \%$ (depending on the specific ginsenoside) of the content in the unprocessed ginseng extract.

The total ginsenoside recovery rate of $67.63 \%$ is consistent with the results of an earlier study that ex- amined the effects of pasteurization $\left(90^{\circ} \mathrm{C}\right.$ for $\left.4 \mathrm{~min}\right)$ of Asian ginseng root, wherein the ginsenosides in the pasteurization mixture constituted $72 \%$ of the ginsenosides in the unpasteurized root material (Jäger et al., 2010). This research team concluded that $P$. ginseng L. root could withstand pasteurization, losing approximately $25 \%$ of the active compounds, and was thus suitable for preparations that involved pasteurization. The difference in ginsenoside content between milk that has been enriched with ginseng extract and unprocessed ginseng extract could thus be due in part to the heat treatment step in the production process. The thermal degradation of ginsenosides has been reported (Choi et al., 1982; Ren and Chen, 1999b), following a first-order kinetic model; further, the rate of degradation can vary substantially depending on the type of ginsenoside and the temperature of the heat treatment (Choi et al., 1982).

Although a thermal lability trend for ginsenosides has not been established, it is possible that the disparate recoveries of individual ginsenosides in the present study can be attributed to their relative sensitivities to heat. Consistent with the report by Corbit et al. (2005), Rg1, $\mathrm{Re}$, and Rb2 had the highest recovery rates, indicating that they are the most thermoresistant ginsenosides; $\mathrm{Rd}$ and Rc, followed by Rb1, had the lowest recoveries, reflecting their status as the most heat-sensitive ginsenosides. Overall, ginsenosides in the ginseng extract had good stability during the UHT treatment, rendering it a suitable ingredient for milk products with cognitionpromoting effects.

In a clinical trial, Scholey et al. (2010) showed that acute oral doses of 100 and $200 \mathrm{mg}$ of ginseng extract (Cereboost), providing 11.6 and $23.3 \mathrm{mg}$ of ginsenosides, respectively, improved cognitive functions 1 , 3 , and $6 \mathrm{~h}$ after consumption. To achieve these doses, the recommended intake of milk enriched with ginseng extract would be between 150 and $300 \mathrm{~mL}$.

\section{Sensory Properties of Milk Enriched with Ginseng Extract}

Mixed ANOVA of sensory data indicated that, for all attributes except viscosity and aftertaste, the effect of the sample was significant $(P<0.05)$. The mean intensity value for each sensory attribute in the milk samples and the significant differences between them are shown in Table 5.

Ginseng extract affected most flavor attributes. The addition of ginseng extract significantly increased the perceived light brown color in the flavored (sample C) and unflavored (sample A) samples, and was highest in the industrial milk with ingredients added before the UHT treatment (sample D). Ginsenosides are known to 

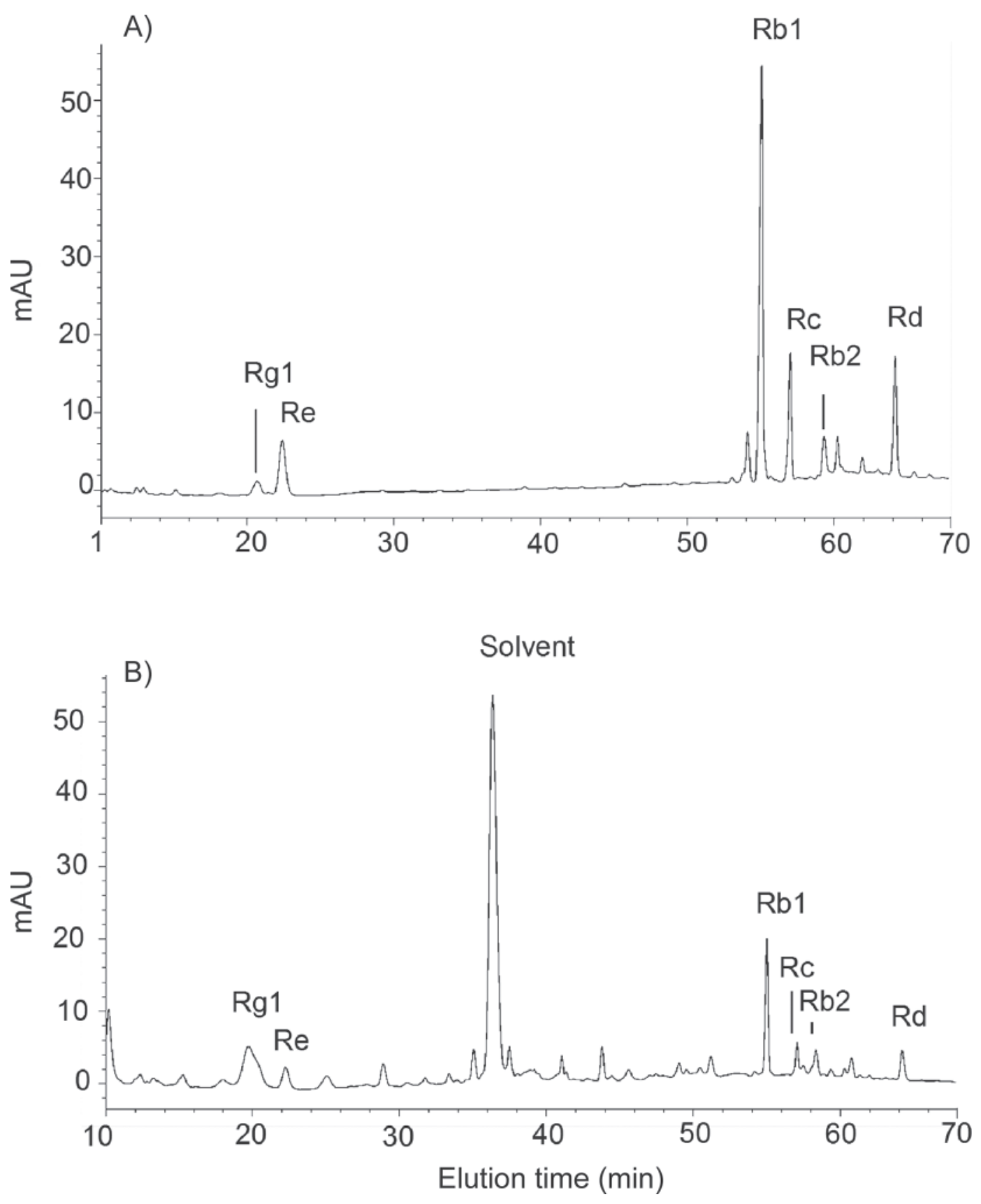

Figure 1. Ginsenoside profiles of A) American ginseng extract (Naturex Inc., South Hackensack, NJ) and B) milk enriched with American ginseng extract.

become deglycosylated during heat processing, and the sugar moieties of ginsenosides are a source of Maillard reaction products with amino acids in ginseng during such treatment (Kang et al., 2007). Although we did not measure the newly formed compounds generated during heat treatment of ginsenosides, it is possible that the browning of ginseng extract-enriched milk resulted from a nonenzymatic Maillard reaction between reducing sugars liberated from the lactose hydrolysis and the amino acids present in the milk, exacerbated by ginsenoside-derived Maillard reaction products. Further studies should be performed to determine the causes of browning that was induced by the ginseng.

As expected, the sweet odor was perceived as significantly $(\alpha=0.05)$ more intense in flavored samples (samples B and C) but decreased slightly in the industrial flavored milk with ingredients added before UHT treatment (sample D). The control sample had 
Table 4. Ginsenoside content in the American ginseng $\operatorname{extract}^{1}(\%)$ and in milk enriched with the American ginseng extract $(\mathrm{mg} / 100 \mathrm{~g}$ of milk), and recovery of ginsenosides $(\%)$

\begin{tabular}{lccc}
\hline Ginsenoside & $\begin{array}{c}\text { Ginsenoside in } \\
\text { ginseng extract }(\%)\end{array}$ & $\begin{array}{c}\text { Ginsenoside in enriched } \\
\text { milk }(\mathrm{mg} / 100 \mathrm{~g} \text { of milk) }\end{array}$ & Recovery $(\%)$ \\
\hline Rg1 & 0.25 & 0.25 & 100.0 \\
Re & 1.76 & 1.52 & 86.1 \\
Rb1 & 5.51 & 3.50 & 63.5 \\
Rc & 1.57 & 0.90 & 57.4 \\
Rb2 & 0.30 & 0.30 & 100.0 \\
Rd & 1.74 & 1.07 & 61.7 \\
Total & 11.12 & 7.53 & 67.6 \\
\hline
\end{tabular}

${ }^{1}$ Cereboost (Naturex Inc., South Hackensack, NJ).

the most intense milk flavor, which dissipated when it was flavored or enriched with ginseng extract (Table 5). The sweetness and intensity of the vanilla flavor of the flavored samples were significantly greater compared with those of the unflavored milk (Table 5). These attributes were less intense in the industrial flavored milk with ingredients added before UHT treatment (sample D) than in the flavored milk (samples B and C); thus, some loss of flavor occurred during the industrial heat treatment. The flash cooling applied in the direct UHT treatment has a deodorizing effect, reducing the content of some volatile compounds in the products (Contarini et al., 1997; Hougaard et al., 2011).

Bitterness was not detected or was very weak in milks without ginseng extract ( 0.4 and 0.8 for the control and sample B, respectively), and it was clearly perceived in samples containing ginseng extract. Between samples $\mathrm{A}$ and $\mathrm{C}$, however, bitterness was lower in the flavored compared with the unflavored sample, indicating that the vanilla aroma and sucralose masked, to some extent, the bitter taste caused by ginseng extract. Heattreatment of the ginseng extract increased the bitterness, with sample D having the most intense bitter taste
(Table 5). The bitterness of ginseng is attributed to the presence of triterpenoid peptides (Court, 2000). The addition of flavors can minimize bitterness, but in our study, the sweetener and vanilla aroma were insufficient to mask the bitter taste. More congruent or related flavors, such as chocolate, citrus, coffee, and cyclodextrins, that form a complex with bitter molecules, as proposed by Tamamoto et al. (2010a), could be more effective in mitigating the bitterness. Ginseng also had a horn effect (an effect that occurs when increased levels of an ingredient decrease the intensity ratings of other attributes; Kappes et al., 2006), whereby the increase in bitterness and metallic taste decreased the intensity of other flavor attributes, such as milk, vanilla, and sweet flavors. Similarly, Tamamoto et al. (2010b) noted that ginseng had a horn effect on sweet and fruity attributes in energy drinks.

Astringency was not detected in control milk, and it increased slightly with the ginseng extract (Table 5), although this increase was significantly greater when the ginseng extract was heat treated (sample D). The metallic/root flavor was significantly higher in samples that were enriched with ginseng extract, especially the

Table 5. Mean values of the intensity scores for the sensory attributes evaluated by the trained panel

\begin{tabular}{|c|c|c|c|c|c|}
\hline Attribute & Control & $\begin{array}{c}\text { Milk with ginseng } \\
\text { extract }^{1} \\
\text { (sample A) }\end{array}$ & $\begin{array}{l}\text { Flavored milk } \\
\text { (sample B) }\end{array}$ & $\begin{array}{l}\text { Flavored milk } \\
\text { with ginseng } \\
\text { extract } \\
\text { (sample C) }\end{array}$ & $\begin{array}{c}\text { UHT-treated } \\
\text { flavored milk } \\
\text { with ginseng extract } \\
\text { (sample D) }\end{array}$ \\
\hline Sweet odor & $0.6^{\mathrm{c}}$ & $1.6^{\mathrm{c}}$ & $7.1^{\mathrm{a}}$ & $6.3^{\mathrm{ab}}$ & $5.1^{\mathrm{b}}$ \\
\hline Milk flavor & $8.1^{\mathrm{a}}$ & $4.8^{\mathrm{b}}$ & $3.8^{\mathrm{bc}}$ & $3.6^{\mathrm{bc}}$ & $2.3^{\mathrm{c}}$ \\
\hline Vanilla flavor & $0.5^{\mathrm{c}}$ & $1.0^{\mathrm{c}}$ & $7.8^{\mathrm{a}}$ & $6.8^{\mathrm{a}}$ & $4.1^{\mathrm{b}}$ \\
\hline Aftertaste $^{2}$ & 2.5 & 3.1 & 2.8 & 3.9 & 3.3 \\
\hline Astringent & $0.7^{\mathrm{c}}$ & $2.3^{\mathrm{b}}$ & $1.3^{\mathrm{bc}}$ & $2.2^{\mathrm{b}}$ & $4.3^{\mathrm{a}}$ \\
\hline Viscosity $^{2}$ & 0.7 & 0.9 & 0.7 & 0.8 & 0.6 \\
\hline
\end{tabular}

\footnotetext{
${ }^{\mathrm{a}-\mathrm{d}}$ For each attribute within a row, values that do not share a superscript letter are significantly different $(\alpha=0.05)$. ${ }^{1}$ Cereboost (Naturex Inc., South Hackensack, NJ).

${ }^{2}$ For these attributes, the effect of the sample was not significant $(\alpha=0.05)$.
} 


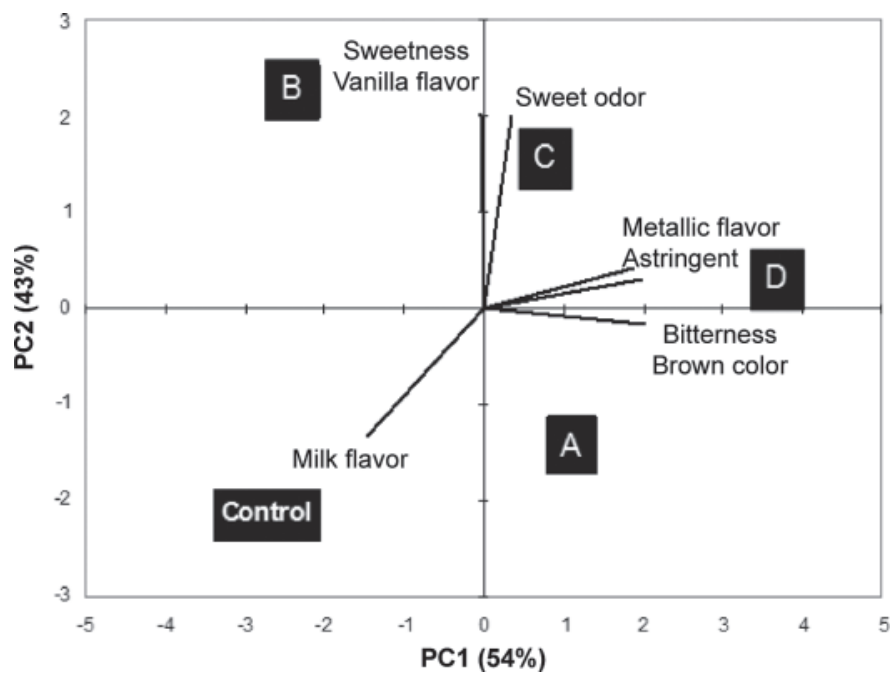

Figure 2. Principal components (PC) analysis plot for sensory properties of the milk samples studied: milk with American ginseng extract (Naturex Inc., South Hackensack, NJ; A), flavored milk (B), flavored milk with American ginseng extract (C), flavored, UHTtreated milk with American ginseng extract (D), and UHT-treated milk (control).

flavored samples (Table 5). All milk samples had low viscosity and aftertaste intensity ratings that did not vary significantly between samples.

To visualize differences in sensory properties among milk samples, PC analysis was applied to the attribute ratings listed in Table 5 (only to those for which the effect of samples was significant). The first 2 components (PC1 and PC2) explained 97\% of the total variance in the data set (Figure 2). Principal component 1, which accounted for $54 \%$ of the total variability, was positively correlated with a metallic flavor, astringency, bitterness, and a brown color. Principal component 2, which accounted for $43 \%$ of the variability, was positively related to sweetness and a vanilla flavor and negatively correlated with a milk flavor.

The position of each sample along the PC axes is displayed in Figure 2. As one would expect, the products containing ginseng (samples A, C, and D) were closer together on the metallic flavor, astringency, bitterness, and brown color (PC1) axis and were separated from the other milks without ginseng (the control and sample B). The effect of ginseng extract on the sensory properties of milk was generally more pronounced in the sample developed in the industrial process, because sample D lay farther away from samples A and C along the $\mathrm{PC} 1$ axis.

The flavored products (samples B, C, and, to a lesser extent, D) were within close proximity on the sweetness and vanilla flavor ( $\mathrm{PC} 2)$ axis, and were farther away from the unflavored samples (sample A and the control). Sample D had a more intense sweetness and vanilla flavor than the unflavored samples but these attributes were less pronounced than those for samples $\mathrm{B}$ and C. These results support the view that heatinduced modifications of ginsenosides may occur, affecting some sensory properties of the product. The intensity of flavors such as sweeteners and the vanilla aroma were decreased by the UHT process.

\section{Consumer Response to the Milk Enriched with Ginseng Extract}

In this exploratory study conducted with 100 participants, we observed that the hedonic response of consumers to samples varied greatly, depending on the interest in the product, especially for the sample enriched with ginseng extract. In this situation, the mean acceptability values may not properly reflect the opinion of the population, and it is recommended that different groups of consumers be studied. In the present work, hedonic responses to the milk samples were grouped for the consumers with different degrees of willingness to consume the product (Table 6). Most of consumers (78\%) indicated that they definitely or probably would consume the highly digestible milk. After tasting the sample, the average product acceptability rating was 7.2 , and $87 \%$ of them indicated that they would buy the sample. When asked about the interest in consuming highly digestible milk enriched with ginseng, $47 \%$ of respondents indicated that were not interested in it, and when they tasted the milk enriched with ginseng, they gave it a low acceptability rating (2.3). However, for the $32 \%$ of consumers that were interested in the milk enriched with ginseng ("Definitely/probably would consume"), the average acceptability value within this group was 6.6, indicating liking of the product, and the majority of consumers in this group (75\%) declared that they would buy it.

In addition to sensory properties, the influence of food habits, attitudes, beliefs, and opinions on the acceptance or rejection of a product could be especially relevant in foods that include a new functional component (Aaron et al., 1994; Urala and Lahteenmaki, 2004; Jaeger, 2006; Villegas et al., 2008). Similar to the result observed in a study carried out to identify ginseng food concepts possessing market potential in the United States (Chung et al., 2011), a proportion of the participants in the present study had a low level of interest in a ginseng product, and for this population, the original ginseng flavors, including bitterness and a metallic/ root flavor, were not considered attractive sensory characteristics. However, for participants who 
Table 6. Acceptability (mean value, SD in parentheses) and purchase intention of the control milk and the UHT-treated milk enriched with American ginseng extract ${ }^{1}$ (sample D) ${ }^{2}$

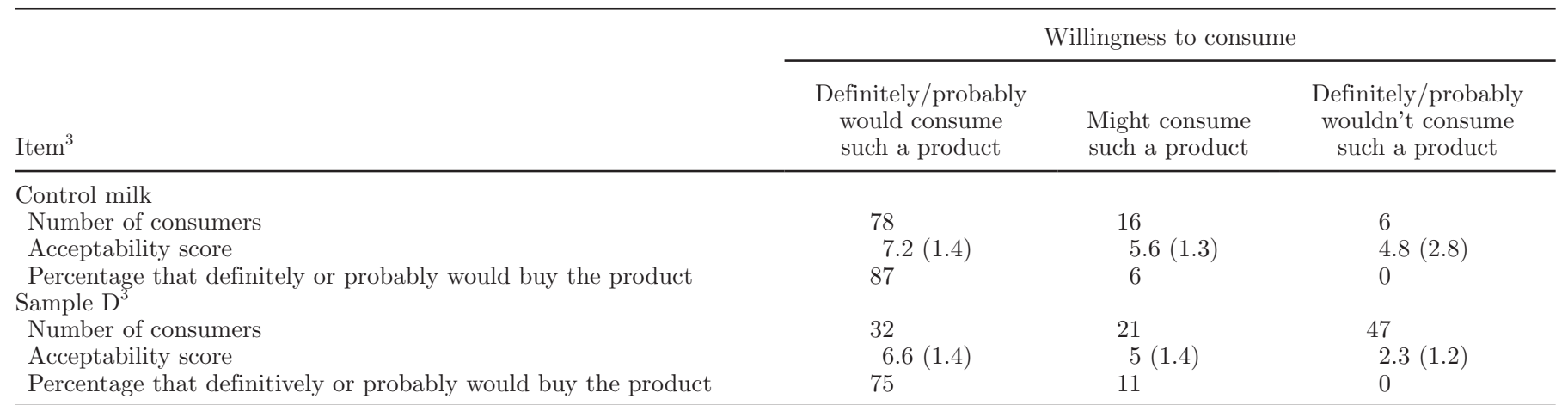

${ }^{1}$ Cereboost (Naturex Inc., South Hackensack, NJ).

${ }^{2}$ Consumer groups were based on the willingness to consume a product with the informed characteristics.

${ }^{3}$ Informed characteristics for control milk: highly digestible semi-skim milk; informed characteristics for sample D: highly digestible semi-skim milk enriched with ginseng extract that improves cognitive function.

were initially more likely to consume the product containing ginseng extract, the acceptability rating was high, indicating that they were more willing to accept these new sensory features provided by the ginseng extract.

\section{CONCLUSIONS}

Developing heat-treated milk with the beneficial health properties of ginseng is feasible. In this study, adding $0.1 \%$ ginseng extract resulted in a beverage enriched with $7.52 \mathrm{mg}$ of ginsenosides/100 g of milk after the UHT process, corresponding to a bioactive recovery of $67.6 \%$. The consumption of 150 to $300 \mathrm{~mL}$ of this ginseng-enriched milk would provide the amount of ginsenosides proven to be effective for improving cognitive functions. This, combined with the low level of lactose, make this drink an appropriate functional beverage for elderly people. The presence of ginsenosides and their thermal treatment affected certain sensory properties of the milk. The most notable changes were the increase in bitterness and metallic taste, the appearance of a brownish color, and a decrease in milky flavor. The presence of flavoring ingredients, such as vanilla and sucralose, decreased the intensity of the bitterness caused by the ginseng extract to some extent. It was found that among consumers within a niche more likely to consume ginseng products, the newly developed milk product was well accepted. Other alternatives should be investigated, such as the addition of more congruent aromas to mask non-milk-related sensory attributes, and the likelihood of consumers accepting unfamiliar flavors to gain increased health benefits from a milk product should be examined.

\section{ACKNOWLEDGMENTS}

The financial support of the Ministry of Science and Innovation of Spain (Madrid, Spain) for the project SENIFOOD (CENIT Programme) and for the contract with A. Tárrega (Juan de la Cierva Programme) is acknowledged. We gratefully acknowledge Juan Duato Aguilar, from Naturex Spain S.L. (Quart de Poblet, Spain), for his valuable technical support.

\section{REFERENCES}

Aaron, J. I., D. J. Mela, and R. E. Evans. 1994. The influences of attitudes, beliefs and label information on perceptions of reduced-fat spread. Appetite 22:25-37.

Attele, A. S., J. A. Wu, and C. S. Yuan. 1999. Ginseng pharmacology: Multiple constituents and multiple actions. Biochem. Pharmacol. 58:1685-1693.

Choi, J. H., D. H. Kim, and H. S. Sung. 1982. Kinetic studies on the thermal degradation of ginsenosides in ginseng extract. Korean J. Food Sci. Technol. 14:197-202.

Christensen, L. P. 2009. Ginsenosides chemistry, biosynthesis, analysis, and potential health effects. Adv. Food Nutr. Res. 55:1-99.

Chung, H. S., H. D. Hong, K. Kim, C. W. Cho, H. R. Moskowitz, and S. Y. Lee. 2011. Consumer attitudes and expectations of ginseng food products assessed by focus groups and conjoint analysis. J. Sens. Stud. 26:346-357.

Contarini, G., M. Povolo, R. Leardi, and P. M. Toppino. 1997. Influence of heat treatment on the volatile compounds of milk. J. Agric. Food Chem. 45:3171-3177.

Corbit, R. M., J. F. S. Ferreira, S. D. Ebbs, and L. L. Murphy. 2005. Simplified extraction of ginsenosides from American ginseng (Panax quinquefolius L.) for high-performance liquid chromatography-ultraviolet analysis. J. Agric. Food Chem. 53:9867-9873.

Court, W. E. 2000. Ginseng: The Genus Panax. CRC Press, Singapore.

González-Tomás, L., S. Bayarri, and E. Costell. 2009. Inulin-enriched dairy desserts: Physicochemical and sensory aspects. J. Dairy Sci. 92:4188-4199.

Harkey, M. R., G. L. Henderson, M. E. Gershwin, J. S. Stern, and R. M. Hackman. 2001. Variability in commercial ginseng products: An analysis of 25 preparations. Am. J. Clin. Nutr. 73:1101-1106. 
Hougaard, A. B., J. S. Vestergaard, C. Varming, W. L. P. Bredie, and R. H. Ipsen. 2011. Composition of volatile compounds in bovine milk heat treated by instant infusion pasteurisation and their correlation to sensory analysis. Int. J. Dairy Technol. 64:34-44.

ISO (International Organization for Standardization). 2007. General Guidance for the Design of Test Room. Standard No. 8589:2007. ISO, Geneva, Switzerland.

Jaeger, S. R. 2006. Non-sensory factors in sensory science research. Food Qual. Prefer. 17:132-144

Jäger, A. K., L. Saaby, D. S. Kudsk, K. C. Witt, and P. Moolgaard. 2010. Short communication: Influence of pasteurization on the active compounds in medicinal plants to be used in dairy products. J. Dairy Sci. 93:2351-2353.

Jia, L., and Y. Zhao. 2009. Current evaluation of the millennium phytomedicine-ginseng (I): Etymology, pharmacognosy, phytochemistry, market and regulations. Curr. Med. Chem. 16:2475-2484.

Kang, K. S., H. Y. Kim, S. H. Baek, H. H. Yoo, J. H. Parkand, and T. Yokozawa. 2007. Study on the hydroxyl radical scavenging activity changes of ginseng and ginsenoside-rb2 by heat processing. Biol. Pharm. Bull. 30:724-728.

Kappes, S. M., S. J. Schmidt, and S. Y. Lee. 2006. Color halo/horns and halo-attribute dumping effects within descriptive analysis of carbonated beverages. J. Food Sci. 71:S590-S595.

Kennedy, D. O., and A. B. Scholey. 2003. Ginseng: Potential for the enhancement of cognitive performance and mood. Pharmacol. Biochem. Behav. 75:687-700

Li, T. S. C., G. Mazza, A. C. Cotrell, and L. Gao. 1996. Ginsenosides in roots and leaves of American ginseng. J. Agric. Food Chem. 44:717-720.

National Sanitation Foundation. 2012. Determination of ginsenosides in Panax ginseng and American ginseng by high-performance liquid chromatography. Panax and American Ginseng Assay by HPLC. INA Method 103.000. National Sanitation Foundation, Ann Arbor, MI. Accessed Jan. 9, 2012. http://www.nsf.org/business/ina/ginseng.asp?program=INA.
Qi, L. W., C. Z. Wang, and C. S. Yuan. 2011. Ginsenosides from American ginseng: Chemical and pharmacological diversity. Phytochemistry 72:689-699.

Ren, G., and F. Chen. 1999a. Simultaneous quantification of ginsenosides in American ginseng (Panax quinquefolium) root powder by visible/near-infrared reflectance spectroscopy. J. Agric. Food Chem. 47:2771-2775.

Ren, G., and F. Chen. 1999b. Simultaneous quantification of ginsenosides in American ginseng (Panax quinquefolium) root powder by visible/near-infrared reflectance spectroscopy. J. Agric. Food Chem. 47:1501-1505.

Scholey, A., A. Ossoukhova, L. Owen, A. Ibarra, A. Pipingas, K. He, M. Roller, and C. Stough. 2010. Effects of American ginseng (Panax quinquefolius) on neurocognitive function: An acute, randomised, double-blind, placebo-controlled, crossover study. Psychopharmacology (Berl.) 212:345-356.

Stone, H., and J. L. Sidel. 1998. Quantitative descriptive analysis: Developments, applications, and the future. Food Technol. J. $52: 48-52$

Sutherland, S. K., S. E. Purdon, C. T. Lai, L. J. Wang, G. Z. Liu, and J. J. Shan. 2010. Memory enhancement from two weeks' exposure to North American ginseng extract HT1001 in young and middle aged healthy adults. Open Nutraceuticals J. 3:20-21.

Tamamoto, L. C., S. J. Schmidt, and S. Lee. 2010a. Sensory properties of ginseng solutions modified by masking agents. J. Food Sci. 75:S341-S347.

Tamamoto, L. C., S. J. Schmidt, and S. Y. Lee. 2010b. Sensory profile of a model energy drink with varying levels of functional ingredients - Caffeine, ginseng, and taurine. J. Food Sci. 75:S271-S278,

Urala, N., and L. Lahteenmaki. 2004. Attitudes behind consumers' willingness to use functional foods. Food Qual. Prefer. 15:793803

Villegas, B., I. Carbonell, and E. Costell. 2008. Effects of product information and consumer attitudes on responses to milk and soybean vanilla beverages. J. Sci. Food Agric. 88:2426-2434. 